\title{
Resolidificación con láser de barreras térmicas de circona depositadas por proyección térmica plasma (APS)
}

\author{
J. C. DIEZ, J. I. PEÑA, V. M. ORERA, M. SIERRA* \\ I.C.M.A. (C.S.I.C.-Univ. Zaragoza), C/María de Luna 3, 50018-Zaragoza \\ * Dpt. Materiales y Procesos ITP, Avda. Castilla. 28830-San Fernando de Henares. Madrid
}

\begin{abstract}
Los recubrimientos de circona parcialmente estabilizada con itria se usan habitualmente como barreras térmicas en turbinas y motores. Estos recubrimientos se suelen realizar mediante proyección térmica plasma (APS), una técnica relativamente económica y bien conocida en el sector industrial. Las barreras consisten de una capa de 0.5 a $1 \mathrm{~mm}$ de cerámica muy porosa unida a la aleación metálica mediante una capa de enlace. La elevada porosidad permite incrementar la resistencia al choque térmico pero provoca que el oxigeno u otros gases perniciosos para el substrato lleguen con relativa facilidad, produciéndose ataques que pueden acelerar el proceso de delaminación. Asimismo la superficie es rugosa y por tanto expuesta a la erosión por partículas. En este trabajo se presentan las condiciones óptimas que permiten la resolidificación mediante láser de la superficie de las capas de Y-PSZ (circona parcialmente estabilizada con itria) depositadas por proyección térmica de plasma en aleaciones de Ni. Se presenta un sencillo modelo que permite explicar la energía umbral de fusión y su dependencia con la velocidad de tratamiento. Se observa cómo la capa externa tratada se densifica, disminuye su rugosidad y queda íntimamente ligada con la capa porosa de Y-PSZ.
\end{abstract}

Palabras clave: Recubrimientos antitérmicos, Recubrimientos de circona, Proyección Térmica Plasma, Fusión con láser.

\section{Laser remelting of plasma sprayed zirconia thermal barrier coatings}

Coatings of partially stabilised zirconia are currently used as thermal barrier coatings in gas turbines and diesel engines. Zirconia coatings are usually processed via plasma spraying a well-established and relatively cheap technique. Thermal barrier coatings consist of 0.5 to $1 \mathrm{~mm}$ of porous ceramic bonded to the metallic substrate by a bond-coat. High porosity in the ceramic coating assure a good thermal behaviour but increases the chances for corrosion or chemical attack of the bondcoat and coating detach by delamination processes. The as sprayed surface is rough and sensitive to erosion. In the present work we obtained the processing conditions for laser remelting of Y-PSZ coatings deposited by atmospheric plasma spraying technique on $\mathrm{Ni}$ - alloys. A simple model accounts for the onset melting energy and its dependence with the processing speed. The remelted film is dense and with smooth surface and well bonded to the underlying porous coat.

Keywords: Thermal barrier coatings, Zirconia coatings, Plasma spraying, Laser melting

\section{INTRODUCCION}

La aplicación de barreras térmicas (TBC's) en álabes de turbinas y motores diesel permite incrementar la temperatura de los gases inyectados en unos 100 a $150^{\circ} \mathrm{C}$ lo que se traduce en un incremento del rendimiento termodinámico de los mismos y en que la temperatura de trabajo de la aleación protegida pueda ser más baja. Con ello se incrementa la vida en funcionamiento de las piezas y se rebaja la necesidad de refrigeración de las mismas (1). Habitualmente una TBC es una capa cerámica porosa que se fabrica mediante la técnica de Proyección Térmica Plasma (APS) sobre la pieza de aleación a proteger intercalando entre las mismas una fina capa de anclaje (BC). Para conseguir un óptimo funcionamiento el material de la TBC ha de cumplir los siguientes requerimientos: a) alto punto de fusión con baja conductividad térmica, b) alta estabilidad térmica frente a transformaciones de fase en el rango entre TA (temperatura ambiente) y la temperatura de trabajo, c) resistente a la fatiga térmica y al choque térmico, d) buena adherencia al substrato metálico y un buen ajuste de su coeficiente de dilatación térmica al de la aleación, e) facilidad de aplicación y baja velocidad de sinterización de su estructura porosa a la temperatura de trabajo $(2,3)$.

De entre los materiales utilizados en las TBC's los más idóneos son la YSZ cúbica, la Y-PSZ y el pirocloro $\mathrm{La}_{2} \mathrm{Zr}_{2} \mathrm{O}_{7}$. Todos ellos son bastante resistentes a la erosión y son compatibles con la $\mathrm{Al}_{2} \mathrm{O}_{3}$ que se forma en la capa de enlace por oxidación. Las TBC's más utilizadas son las de circona parcialmente estabilizada con un $3 \%$ mol de $\mathrm{Y}_{2} \mathrm{O}_{3}$. La fase tetragonal de la circona es muy resistente a la propagación de grietas debido al efecto de tenacidad por transformación característico de esta fase metaestable de la circona. Asimismo el material es un gran aislante térmico y muy estable y resistente hasta alta T. Los recubrimientos se producen proyectando el material fundido por un haz de plasma sobre la aleación base en la que previamente se depositó una capa de anclaje de material antioxidante. Así se produce una capa altamente texturada en la dirección perpendicular a la superficie de deposición y muy porosa. La presencia de poros contribuye por un lado a mejorar las características aislantes del recubrimiento y su resistencia al agrietamiento pero inevitablemente aumenta la posibilidad de que aparezcan redes de grietas y porosidad conectada que favorece la corrosión a alta T de la capa (4). En efecto, la principal causa de fallo de las TBC's parece ser la debida a la oxidación y corrosión de la BC. En particular la PSZ es atacada por el vapor de agua a altas T. Además, la conductividad térmica y la resistencia al choque térmico de la capa están relacionadas con su morfología, de forma que a menor porosidad mayor módulo 
pero también mayor conductividad. También la resistencia a los ciclos térmicos depende de la porosidad y de la distribución de grietas en el material.

Una posible solución que permita mantener la resistencia a los choques térmicos del material poroso y a la vez evitar la corrosión y eliminar rugosidad de la superficie del recubrimiento lo que mejoraría su comportamiento frente a erosión (5), consiste en realizar una resolidificación del recubrimiento en superficie utilizando la técnica de fusión por láser. Esencialmente, la técnica consiste en aplicar un haz láser de suficiente intensidad como para fundir la superficie del material mientras que la pieza se desplaza relativamente a este con objeto de tratar toda la superficie. Las ventajas de la técnica láser son la limpieza, acceso con el haz a cualquier parte de la superficie y que al ser un tratamiento local no perturba al resto del material. Tanto la técnica de deposición por proyección de plasma como la de resolidificación láser pueden aplicarse "in situ". La técnica de resolidificación mediante láser de recubrimientos cerámicos ha sido objeto de diversos estudios $(6,7)$. En general, se utiliza un láser de $\mathrm{CO}_{2}$ expandido hasta formar un haz rectangular y se realiza un precalentamiento de las piezas para tratar de evitar el agrietamiento que se produce debido a la contracción de la capa fundida.

En ésta investigación se trata de demostrar la capacidad de la técnica de la resolidificación para sellar porosidad y producir una superficie lisa pero utilizando un láser de diodos en vez de uno de $\mathrm{CO}_{2}$ en una TBC de circona depositada por APS. También se trata de obtener valores de parámetros de tratamiento tales como potencia umbral, espesores, etc. y estimaciones de rendimientos.

\section{MATERIALES Y DISPOSITIVOS EXPERIMENTALES}

Los substratos utilizados en esta investigación fueron placas de superaleación base $\mathrm{Ni}$ (Hastelloy $X^{\circledR}$ ). Las placas fueron granalladas con polvo de alúmina en aire y luego se les depositó mediante la técnica de proyección de plasma en aire una capa de anclaje de aleación NiCrAlY de unas $100 \mu \mathrm{m}$ de espesor. Posteriormente se les proyectó una capa de circona parcialmente estabilizada con itria Y-PSZ $\left(3 \mathrm{~mol} \% \mathrm{Y}_{2} \mathrm{O}_{3}\right)$ consiguiéndose una TBC de unas $300 \mu \mathrm{m}$ de espesor. Es importante hacer notar que la capa de circona presenta una coloración grisácea. En la tabla I se indican los parámetros de proyección empleados para depositar la capa de anclaje y el recubrimiento de Y-PSZ.

Para conseguir densificar y fundir la capa superficial de la TBC, las muestras se trataron con un láser continuo de diodos Rofin \& Sinar $(\lambda=808 \mathrm{~nm})$ de $250 \mathrm{~W}$ de máxima potencia en el que se dispusieron dos lentes cilíndricas ortogonales con objeto de obtener un haz homogéneo y rectangular de aproximadamente $10 \times 1 \mathrm{~mm}^{2}$ de sección sobre la superficie a tratar. En este caso no se utilizó precalentamiento de las muestras. La potencia del láser fue medida utilizando un divisor de haz y un medidor termoeléctrico. Las muestras se procesaron con las velocidades de desplazamiento del haz de 1500, 2000, 2500, $3000 \mathrm{~mm} / \mathrm{h}$ disponibles en el laboratorio que corresponden a rendimientos de $0.015,0.02,0.025$ y $0.03 \mathrm{~m}^{2} / \mathrm{h}$. Hay que hacer notar que estos rendimientos corresponden a una instalación de laboratorio y que pueden ser aumentados en dos ordenes de magnitud empleando láseres industriales.

La estructura cristalina de la TBC antes y después del tratamiento con láser fue estudiada usando la espectroscopia Raman con un espectrómetro DILOR X-Y dotado de microson-
Tabla I. Parámetros empleados en el proceso de Proyección TÉRMICA Plasma

\begin{tabular}{|c|c|c|c|}
\hline Capa & Anclaje & & Y-PSZ \\
\hline Tipo de polvo: denominación comercial & \multicolumn{2}{|c|}{ AMDRY 962F } & M204NS \\
\hline Descripción, composición química & \multicolumn{2}{|c|}{ Ni22Cr10Al1Y } & $\mathrm{ZrO} 2-8 \% \mathrm{Y} 2 \mathrm{O} 3$ \\
\hline \multicolumn{4}{|l|}{ Especificaciones } \\
\hline Pistola plasma. Nombre Comercial & & SM-F100 & SM-F100 \\
\hline Diámetro electrodo & $\mathrm{mm}$ & 11 & 11 \\
\hline Diámetro inyector & $\mathrm{mm}$ & 1.8 & 1.8 \\
\hline Ángulo inyector & ${ }^{\circ}$ & 90 & 90 \\
\hline Corriente & A & 350 & 400 \\
\hline Voltaje & V & 39 & 42 \\
\hline Gas primario: Argon (Ar) & \multirow{2}{*}{$\begin{array}{l}\text { slpm } \\
\text { slpm }\end{array}$} & \multirow{2}{*}{\begin{tabular}{|l|}
45 \\
5 \\
\end{tabular}} & 30 \\
\hline Gas secundario: Hidrogeno $\left(\mathrm{H}_{2}\right)$ & & & 4 \\
\hline Gas portador $(\mathrm{Ar})$ & slpm & 4 & 4.0 \\
\hline Caudal alimentación de polvo & $\mathrm{g} / \mathrm{min}$ & 40 & 60 \\
\hline Velocidad rotación disco & $\%$ RPM & 20 & 40.5 \\
\hline Velocidad rotación agitador & RPM & 60 & 60 \\
\hline Distancia: pistola/pieza & $\mathrm{mm}$ & 70 & 70 \\
\hline Velocidad superficie & $\mathrm{m} / \mathrm{min}$ & 75 & 75 \\
\hline
\end{tabular}

da. La rugosidad de las capas fue determinada por perfilometría (Dektak3ST). Se hicieron barridos de $20 \mathrm{~mm}$ de longitud y se determinó la rugosidad mediante la media aritmética $R_{a}$ conocida formalmente como CLA.

La caracterización de la microestructura de las muestras se realizó utilizando métodos de análisis mediante microscopía óptica para lo que las muestras fueron pulidas utilizando métodos metalográficos estandar. Para estudiar la evolución de la microestructura con el tratamiento las muestras fueron cortadas transversalmente con una sierra de diamante en medio aceitoso, e infiltradas con resina epoxi. Luego fueron desbastadas y pulidas con polvo de diamante hasta $0.25 \mu \mathrm{m}$.

\section{RESULTADOS Y DISCUSIÓN}

En la figura 1 se aprecia la morfología de las TBC de circona después de ser proyectada. Se observa una estructura de microgrietas formando una red con tendencia a situarse paralela a la superficie y con grandes agregaciones de poros interconectados como debe de corresponder a la morfología de una capa formada por granos proyectados en caliente contra un blanco.

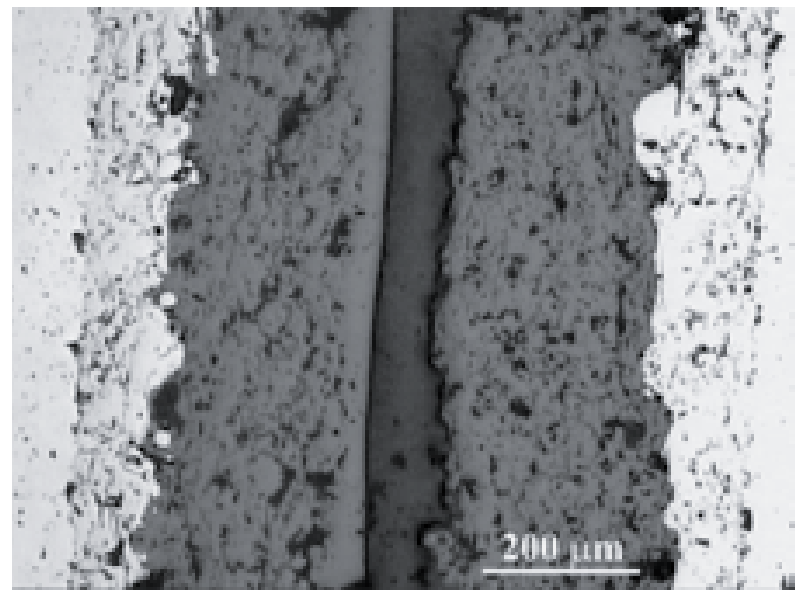

Fig. 1 Micrografía óptica en la que se observan dos cortes transversales. A la derecha el de una capa sin tratar pudiendo verse el substrato, la capa de anclaje y la TBC de circona. A la izquierda la capa tratada con $175 \mathrm{~W}$ a $2500 \mathrm{~mm} / \mathrm{h}$ en la que se aprecia la zona refundida, que tiene aproximadamente unas $40 \mu \mathrm{m}$ de espesor. 


\subsection{Procesado láser}

El tratamiento con láser a distintas potencias produce los siguientes cambios en la morfología de las capas:

I) A bajas potencias de láser se produce un blanqueo de la muestra que pasa del color gris inicial al blanco. El blanqueo de la circona por el láser ha sido ya observado anteriormente (4). La posible explicación de este fenómeno radica en la destrucción de los centros de color producidos por las condiciones reductoras existentes durante la fabricación de la capa y que dan lugar a la coloración de la misma $(8,9)$. El blanqueo de los centros de color se produce durante la reoxidación que tiene lugar durante el calentamiento en aire. Es importante hacer notar que en el caso de un láser de diodos, la presencia de estos centros de color ayuda a la absorción de la radiación láser por la muestra.

II) Cuando se alcanza una determinada potencia, potencia umbral $\mathrm{P}_{\mathrm{m}^{\prime}}$ el material funde. En la figura 2 representamos la potencia umbral, entendida como la densidad de potencia por unidad de superficie a la que se empieza a observar la fusión de la capa, en función de la velocidad de procesado "v". En la gráfica se obtiene una dependencia lineal entre esta potencia umbral y la raíz cuadrada de la velocidad de procesado de la forma:

$$
\mathrm{P}_{\mathrm{m}}=\mathrm{P}_{0}+\mathrm{bv}^{1 / 2}
$$

con $\mathrm{P}_{0}=800 \mathrm{Wcm}^{-2} \mathrm{y} \mathrm{b}=3500 \mathrm{Ws}^{1 / 2} \mathrm{~cm}^{-5 / 2}$.

Para obtener información sobre el significado físico de la ecuación (1) vamos a utilizar un modelo simple que dé cuenta del fenómeno de fusión. En primer lugar hemos de considerar que al incidir sobre la muestra parte del haz láser se refleja y parte se absorbe. La fracción de energía absorbida depende de las constantes ópticas del recubrimiento, siendo mayor para recubrimientos de color obscuro que absorben en la longitud de onda de emisión del láser de diodos. Los recubrimientos, de color gris oscuro, tienen muy poca reflectancia a baja temperatura. El blanqueo de las muestras inducido por el procesado láser hace aumentar la reflectancia hasta un 30\%. A alta T la reflectancia se reduce grandemente debido al incremento en la densidad de fonones que incrementa la absorción por lo que en una primera aproximación podemos despreciar la pérdida de radiación por reflexión. En segundo lugar es evidente que en estático la fusión tiene lugar cuando se absorbe una densidad de potencia que es superior al balance de las pérdidas por radiación, convección y difusión y la muestra alcanza su temperatura de fusión $2973 \mathrm{~K}$. Tratemos de calcular estas pérdidas:

Las pérdidas por radiación vienen dadas por la ley de Stefan-Boltzman $\sigma \mathrm{T}^{4}$ con $\sigma=5.7 \times 10^{-12} \mathrm{WK}^{-4} \mathrm{~cm}^{-2}$ y pueden estimarse teniendo en cuenta la temperatura de fusión y la emisividad de la circona fundida $\varepsilon$. No disponemos del valor de la emisividad de la circona fundida pero podemos tomar un valor de 0.9 por comparación con la de otros óxidos fundidos (10).

$$
\mathrm{P}_{\mathrm{r}}=\varepsilon \sigma \mathrm{T}^{4} \approx 400 \mathrm{Wcm}^{-2}
$$

Las pérdidas por conducción se pueden estimar utilizando una aproximación unidimensional al problema mediante la formula

$$
\mathrm{P}_{\mathrm{d}}=\kappa(\delta \mathrm{T} / \delta \mathrm{x})
$$

Utilizando un valor para la conductividad térmica de $\kappa$ $=0.02 \mathrm{Wcm}^{-1} \mathrm{~K}^{-1}$ a $1000{ }^{\circ} \mathrm{C}$ y considerando que el substrato se calienta a unos $200{ }^{\circ} \mathrm{C}$ y que el espesor del recubrimiento es de

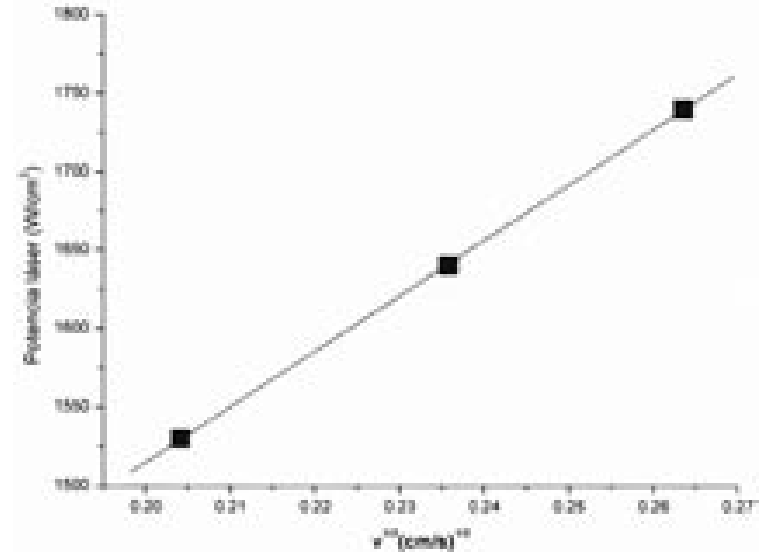

Fig. 2. Representación de la potencia del láser con la que se inicia la fusión (Potencia umbral) en relación con la velocidad de tratamiento.

unas $500 \mu \mathrm{m}$ resulta $\mathrm{P}_{\mathrm{d}} \approx 1000 \mathrm{Wcm}^{-2}$. La suma de $\mathrm{P}_{\mathrm{r}}+\mathrm{P}_{\mathrm{d}}$ es del orden de la experimental pero un poco mayor. Las diferentes causas que pueden contribuir a esta discrepancia van desde el error en el ajuste a la curva experimental con solo tres datos y bastante próximos en energía hasta la simplicidad del modelo e incluso la sobrevaloración de la conductividad térmica que para una capa porosa será algo menor que la del material masivo utilizada aquí.

La contribución dinámica a la ecuación (1) descrita por el parámetro " $b$ " y por la dependencia de la potencia umbral con $\mathrm{v}^{1 / 2}$ puede deducirse de la solución de la ecuación que describe la difusión del calor cuando el láser incide sobre un substrato semiinfinito irradiado uniformemente por un pulso de láser de duración $\tau_{1}(11)$. Haciendo la analogía entre un barrido del láser y un pulso de duración $\tau_{1}=\delta / \mathrm{v}$ donde $\delta$ es la anchura del haz rectangular podemos utilizar la solución de la ecuación de calor en la que suponiendo una superficie de irradiación grande sobre la que incide una densidad de flujo radiativo $\phi=$ $\mathrm{P} \tau_{1}$ que se absorbe en la superficie debido al alto coeficiente de absorción se llega a la siguiente expresión para el flujo necesario para fundir la superficie (11):

$$
\phi_{\mathrm{m}}=(\sqrt{ } \pi / 2) \kappa \theta\left(\tau_{1} / \mathrm{D}\right)^{1 / 2}
$$

donde $\theta$ es la diferencia de temperatura entre el punto fundido $\mathrm{y}$ un punto del substrato muy alejado $\left(\mathrm{T}_{\mathrm{amb}}\right), \kappa$ es la conductividad térmica y $\mathrm{D}$ la difusividad térmica que para la $3 Y-P S Z$ es $0.58 \times 10^{-2} \mathrm{~cm}^{2} \mathrm{~s}^{-1}$ a $1000{ }^{\circ} \mathrm{C}(2)$. Partiendo de esta expresión podemos obtener el valor de la potencia umbral de fusión como:

$$
\mathrm{P}=\sqrt{ } \pi \kappa \theta(1 / 4 \delta \mathrm{D})^{1 / 2} \mathrm{v}^{1 / 2}=\mathrm{bv}^{1 / 2}
$$

siendo $\mathrm{b}=2000 \mathrm{Ws}^{1 / 2} \mathrm{~cm}^{-5 / 2}$ que es del orden del valor experimental pero menor. Habida cuenta de la simplicidad del modelo el resultado es bueno, y puede ser utilizado para predecir los parámetros de procesado a escala industrial. El modelo permite dar una explicación del efecto del procesado.

\subsection{Microestructura}

El procesado en estas condiciones produce una capa densa y suave como se puede observar en las figuras 1 y 3 . La rugosidad promedio tras el proceso de resolidificación se reduce en más de un orden de magnitud. Se obtuvieron valores de Ra $=2.65 \pm 0.85 \mu \mathrm{m}$ en las superficies sin tratar y de $\mathrm{Ra}=0.22 \pm$ $0.06 \mu \mathrm{m}$ en las tratadas. 
Mediante espectroscopía Raman se ha observado que la capa resolidificada presenta la misma estructura cristalina tetragonal que la capa inicial. La superficie resolidificada presenta una estructura de granos poligonales de unas $100 \mu \mathrm{m}$ de diámetro con fronteras de grano formadas por segmentos rectos que concurren en puntos triples (Fig. 4). La diferencia de densidad entre la capa proyectada y resolidificada, que es consecuencia de las diferencias en porosidad existentes entre ambas capas, así como las tensiones termoelásticas producidas por la diferencia en coeficientes de dilatación entre el material denso y la capa porosa y por los enormes gradientes térmicos producidos en el tratamiento (varias veces superiores a las condiciones en servicio), se acomodan mediante la producción de grietas transversales, que en la superficie siguen un patrón de propagación a través de las juntas de grano, tal y como se puede observar con toda claridad en la figura 4.

Podría parecer a primera vista que estas grietas fueran muy perniciosas para las propiedades finales, pero tal y como se puede observar en la figura 3, todas ellas se limitan a la capa resolidificada y son rápidamente paradas por la porosidad preexistente en la capa resultante de la proyección térmica. La limitación de las grietas transversales a la zona fundida se observa en todas las grietas, independientemente de su grosor. Además la presencia de grietas transversales no favorecen la delaminación de la capa y pueden ser incluso beneficiosas para incrementar la resistencia de la misma a los choques térmicos. Por otra parte, y en el caso de que estas grietas pudieran constituir un problema, se pueden cerrar con una segunda resolidificación aportando material, tal como ha sido reportado por G. Antou et al (4).

En vista de los resultados de la microestructura resultante, se puede esperar que estas barreras térmicas resolidificadas presenten unas propiedades que sumen las ventajas de las barreras térmicas producidas mediante técnicas de proyección de plasma (baja conductividad térmica debido a la alta porosidad) con las barreras térmicas obtenidas mediante técnicas de haz de electrones (alta resistencia a gases y oxidación debido a la baja porosidad).

\section{RENDIMIENTOS}

Utilizando la ecuación (5) podemos extrapolar un rendimiento en una instalación industrial típica. Utilizando un láser de $1 \mathrm{~kW}$ enfocado en una línea de $10 \times 1 \mathrm{~mm}^{2}$ obtenemos velocidades de tratamiento de $25 \mathrm{~cm}^{2} / \mathrm{s}$ que corresponden con rendimientos de $9 \mathrm{~m}^{2} / \mathrm{h}$ muy competitivos a nivel industrial.

\section{CONCLUSIONES}

Como conclusión se puede decir que la técnica de resolidificación de una capa cerámica de barrera térmica de Y-PSZ mediante un haz láser en forma de línea procedente de un láser de diodos, permite formar una fina capa de material denso que prácticamente elimina la conexión entre la capa de enlace y el exterior. La capa tratada queda bien adherida al substrato y presenta una superficie lisa. El procedimiento puede escalarse a nivel industrial con buenos rendimientos. Quedan por realizar experimentos de ciclado térmico y corrosión para corroborar que el procesado propuesto supone una ventaja de cara a la mayor duración de la capa en funcionamiento.

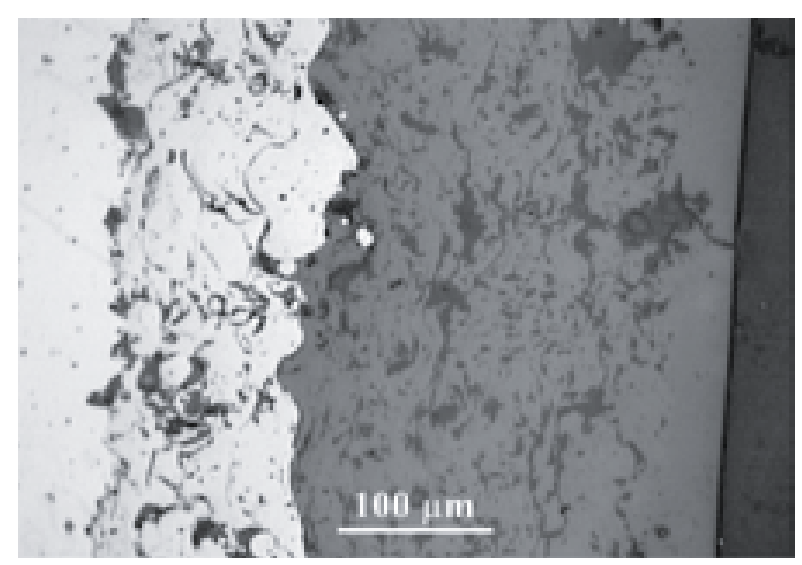

Fig. 3 Detalle de capa tratada con el láser.

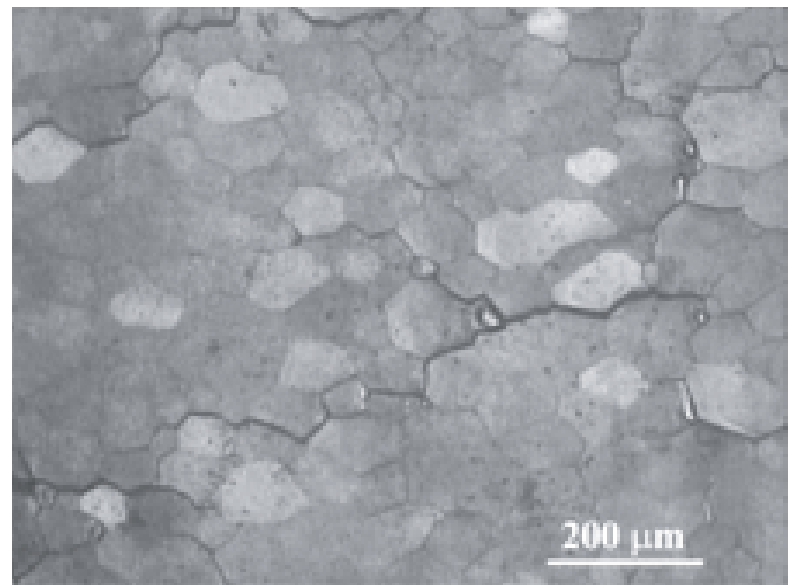

Fig. 4. Micrografía que muestra la superficie de un recubrimiento de circona después de haber sido refundida con el láser.

\section{AGRADECIMIENTOS}

Este proyecto ha sido financiado por el MCyT a través del proyecto MAT2003-06085-C03-01

\section{BIBLIOGRAFÍA}

1. D. Stoever, C. Funke. "Directions of the development of thermal barrier coatings in energy applications". J. Mater. Process. Technol., 92-93, 195-202 (1999)

2. X.Q. Cao, R. Vassen, D. Stoever, " Ceramic materials fot thermal barrier coatings". J. Europ. Ceram. Soc. 24, 1-10 (2004)

3. D.R. Clark. "Materials selection guidelines for low thermal conductivity thermal barrier coatings". Surf. Coat. Technol., 163-164, 67-74 (2003)

4. G. Antou, G. Montavon, F. Hlawka, A. Cornet, C. Coddet, F. Machi. “Modification of ceramic thermal spray deposit microstructures implementing in situ laser remelting". Surf. Coat. Technol., 172, 279-290 (2003)

5. X. Chen, M.Y. He, I. Spitsberg, N.A. Fleck, J.W. Hutchinson, A.G. Evans. "Mechanism governing the high temperature erosion of thermal barrier coatings". Wear, 256, 735-746 (2004)

6. K.C. Chang, W.J. Wei, C. Chen. "Oxidation behavior of thermal barrier coatings modified by laser remelting". Surf. Coat. Technol., 102, 197-204 (1998)

7. Y. Yuanzheng, Z. Youlan, L. Zhengyl, C. Yuzhi. " Laser remelting of plasma sprayed $\mathrm{Al}_{2} \mathrm{O}_{3}$ ceramic coatings and subsequent wear resistance". Mater. Sci. Eng., Part A, 291, 168-72 (2000)

8. V.M. Orera, R.I. Merino, Y. Chen, R. Cases, P.J. Alonso. “Intrinsic electron and hole defects in stabilized zirconia single crystals". Phys. Rev. B, 42, 97829789 (1990)

9. V.M. Orera, R.I. Merino, Y. Chen, R. Cases, P.J. Alonso. “Electron and hole trapped defects produced by thermo-reduction or irradiation in stabilized zirconia “. Rad. Eff. Def. Sol., 119-121, 907-912 (1991)

10. D.O. Nason, C.T. Yen, W.A. Tiller. "Measurements of optical properties of some molten oxides" J. Cryst. Growth 106, 221-226 (1990)

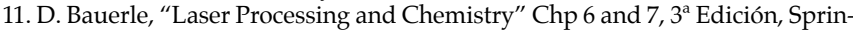
ger-Verlag (2000), ISBN 3-540-66891-8 